\title{
Potential zum Standardwerk
}

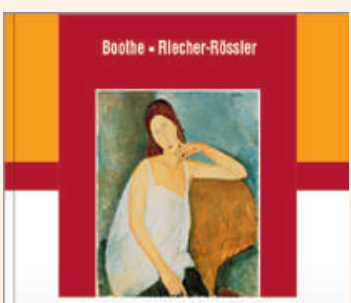

Frauen in Psychotherapie

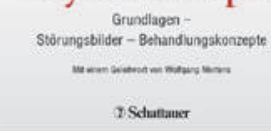

Brigitte Boothe,

Anita Riecher-Rössler (Hrsg.) Frauen in Psychotherapie

Grundlagen - Störungsbilder Behandlungskonzepte Stuttgart: Schattauer; 2013. 524 Seiten, 22 Abb.,

17 Tab., 79 CHF. ISBN 978-3-7945-2814-1

Korrespondenz:

Dr. med. Markus Binswanger Facharzt für Psychiatrie und Psychotherapie Obere Bahnhofstrasse 9 CH-9500 Wil
«Frauen in Psychotherapie», dieses von zwei sehr renommierten Forscherinnen herausgegebene Werk ist eine umfassende Bestandsaufnahme einer Thematik, die bislang selten umfangreich bearbeitet worden ist.» So leitet Wolfgang Mertens das vorliegende CEuvre mit mehr als 500 Seiten und 42 Kapiteln aus der Feder berufener Autorinnen und Autoren ein. In der Tat ist es Brigitte Boothe, Psychoanalytikerin und emeritierte Professorin für Psychologie, sowie Anita Riecher-Rössler, Psychotherapeutin, Chefärztin und Ordinaria für Psychiatrie, gelungen, in diesem breit gefächerten Band fundiertes genderbezogenes psychiatrisches, psychotherapeutisches und psychosomatisches Fachwissen zu integrieren. Die besondere editorische Leistung erschliesst sich daraus, dass - trotz der Vielzahl von Beiträgen aus ganz unterschiedlichen Blickwinkeln das vorgelegte Werk als «aus einem Guss» imponiert.

Der erste Teil ist den Grundlagen sowie der Bedeutung der Genderperspektive gewidmet. Er zeigt auf, dass sich Psychotherapie als Profession zunehmend feminisiert. Zudem wird differenziert erläutert, wie sich die Lebensbedingungen für Frauen in der spätmodernen Lebenswelt in rasantem Tempo verändern, was sowohl zahlreiche Herausforderungen als auch Risiken für die seelische Gesundheit mit sich bringt. Psychische Vulnerabilität wird so im Kontext neuer Sozialisationsmuster, neuer Bindungsformen und neuer Berufskarrieren sowie auch unter kulturspezifischen Aspekten von Globalisierung und Migration beleuchtet. Vor diesem Hintergrund werden frauenspezifische Belastungen in unterschiedlichen Lebensbereichen identifiziert - so in Partnerschaft und Trennung, in Schwangerschaft und Postpartalzeit, bei Verlust eines Kindes durch Todgeburt, bei körperlicher Beeinträchtigung sowie im Rahmen häuslicher Gewalt.

Im umfangreichen zweiten Buchteil gelangen störungsspezifische Behandlungskonzepte für eine Grosszahl von Krankheitsbildern zur Darstellung, so namentlich für die bei Frauen sehr viel häufiger vorkommenden Ess- und Affektstörungen sowie auch für Problemstellungen im Zusammenhang mit Kinderlosigkeit, Schwangerschaft, Geburt, Perimenopause und im Alter. Eine genderspezifische therapeutische Perspektive auf Internetnutzung, kosmetische Chirurgie und forensische Fragestellungen ergänzt diese praxisnahen Kapitel. Schliesslich folgen genderbezogene Beschreibungen der wichtigsten Psychotherapieverfahren und -settings. Erfreulich ist der Umstand, dass trotz der psychodynamischen Orientierung der beiden Herausgeberinnen keine therapeutische Schule bevorzugt und stattdessen der interdisziplinäre Dialog zwischen den einzelnen Verfahren gesucht und gepflegt wird. Bemängelt werden könnte allenfalls die fehlende Darstellung der Behandlung schwerster psychiatrischer Erkrankungen wie Bipolare Störung, Schizophrenie sowie Demenz.

\section{Eine umfassende Bestandsaufnahme.}

Der dritte und letzte Buchteil ist allgemeinen Fragestellungen zu Forschung, Versorgung und guter psychotherapeutischer Praxis gewidmet. In diesem Rahmen erhalten die Themen Missbrauch in der Therapie und traumazentrierte Behandlungen ihren verdienten Stellenwert. Das genderspezifische «Opus magnum» wird durch einen wertvollen Artikel abgeschlossen, der wichtige Frauenfragen auf dem Hintergrund von französischer Revolution sowie Aufklärung beleuchtet und $\mathrm{zu}$ «Intelligenz und Mut in der Genderperspektive» auffordert.

Alle Kapitel können problemlos für sich alleine gelesen werden. Sie sind didaktisch geschickt aufgebaut und durch prägnante Zwischentitel, hilfreiche Querverweise sowie ausführliche Literaturlisten strukturiert. Ein kompaktes Fazit am Schluss jedes Kapitels erhöht den Lesegenuss. Den Verantwortlichen ist es ein Anliegen, angesichts der Fülle der dargestellten Themen die Leserinnen und Leser nicht zu überfordern, sondern praxisbezogen Orientierungshilfen für Diagnostik und Therapie leidender Patientinnen anzubieten.

Was das Werk besonders auszeichnet ist eine auf wissenschaftliche Basis und neueste Forschungserkenntnisse rekurrierende psychotherapeutische Praxis. Dies erlaubt den Autorinnen und Autoren eine sachliche, unaufgeregte Haltung auch gegenüber teilweise sehr emotionalen Frage- und Problemstellungen. Die wichtige feministische Frauenbewegung der 70er Jahre sowie die nachfolgenden gendertheoretischen Diskurse werden systematisch dargestellt - ohne $\mathrm{zu}$ polarisieren oder sich in kontraproduktiven Genderideologien zu verlieren.

Das Buch «Frauen in Psychotherapie» verdient Resonanz. Es verfügt über das Potential, im Verlauf weiterer Auflagen zu einem Standardwerk zu werden. Dieses kann dann - so bleibt zu hoffen - als Modell für ein ähnlich umfassendes und gelungenes Werk dienen - nämlich über «Männer in Psychotherapie». 